Roger Moser

\title{
The inverse mean curvature flow and $p$-harmonic functions
}

Received October 12, 2005

Abstract. We consider the level set formulation of the inverse mean curvature flow. We establish a connection to the problem of $p$-harmonic functions and give a new proof for the existence of weak solutions.

\section{The problem}

For $n \geq 2$, let $\Omega \subset \mathbb{R}^{n}$ be an open set with smooth boundary such that its complement, $\Omega^{c}=\mathbb{R}^{n} \backslash \Omega$, is bounded. We study the problem

$$
\begin{array}{r}
\operatorname{div}\left(\frac{\nabla u}{|\nabla u|}\right)=|\nabla u| \quad \text { in } \Omega, \\
u=0 \quad \text { on } \partial \Omega .
\end{array}
$$

This can be regarded as a level set formulation of a parabolic evolution problem for hypersurfaces in $\mathbb{R}^{n}$ : Suppose $F: M^{n-1} \times[0, T) \rightarrow \mathbb{R}^{n}$ is a family of embedded hypersurfaces evolving by

$$
\frac{\partial F}{\partial t}=-\frac{H}{|H|^{2}}
$$

where $H$ is the mean curvature vector of $M_{t}=F(M, t)$ (with a sign convention such that round spheres expand under the flow). If a function $u: \Omega \rightarrow[0, \infty)$ exists on a certain open set $\Omega \subset \mathbb{R}^{n}$, such that $u \equiv t$ on $M_{t}$, and if this $u$ is sufficiently smooth and satisfies $\nabla u \neq 0$, then it is a solution of (1). If, in addition, $\partial \Omega \subset M_{0}$, then (2) is satisfied as well.

This evolution problem is called the inverse mean curvature flow. It has been studied by Gerhardt [1], Urbas [10], Huisken-Ilmanen [3, 2, 4, 5], Smoczyk [8], and others. The inverse mean curvature flow (on other manifolds than $\mathbb{R}^{n}$ ) has been used by HuiskenIlmanen [3, 4] to prove the Riemannian Penrose inequality from general relativity. Moreover, a theory of weak solutions of (1) was developed in [3, 4], based on a variational principle involving the functionals

$$
J_{u}(w ; K)=\int_{K}(|\nabla w|+w|\nabla u|) d x
$$

for precompact sets $K \subset \Omega$.

R. Moser: Department of Mathematical Sciences, University of Bath, Bath BA2 7AY, United Kingdom; e-mail: r.moser@maths.bath.ac.uk 
Definition 1.1. A function $u \in C_{\mathrm{loc}}^{0,1}(\Omega)$ is called a weak solution of 1 if for every precompact set $K \subset \Omega$ and every $w \in C_{\mathrm{loc}}^{0,1}(\Omega)$ with $w=u$ in $\Omega \backslash K$, the inequality

$$
J_{u}(u ; K) \leq J_{u}(w ; K)
$$

holds. A weak solution is proper if

$$
\lim _{|x| \rightarrow \infty} u=\infty
$$

One of the main results in [4] is an existence result: For every $\Omega \subset \mathbb{R}^{n}$ as above, a proper weak solution $u \in C_{\mathrm{loc}}^{0,1}(\bar{\Omega})$ of $(1)$ and $(2)$ exists. Moreover, proper weak solutions of the problem are unique. We give another proof of the existence result in this paper with a completely different method. Our approach is based on an approximation of (1) by the equations

$$
\operatorname{div}\left(|\nabla u|^{p-2} \nabla u\right)=|\nabla u|^{p} \quad \text { in } \Omega
$$

for $p>1$. We use the following observation: If

$$
v=\exp \left(\frac{u}{1-p}\right),
$$

then (4) is equivalent to

$$
\operatorname{div}\left(|\nabla v|^{p-2} \nabla v\right)=0 \quad \text { in } \Omega .
$$

This, in contrast to (1), is the Euler-Lagrange equation of a variational problem, even a rather simple one. It is no problem at all to find a function in the homogeneous Sobolev space $\dot{W}^{1, p}\left(\mathbb{R}^{n}\right)$ that solves $\sqrt{5}$ in $\Omega$ and satisfies $v=1$ in $\Omega^{c}$. If we can find a limit of such solutions for $p \rightarrow 1$, this limit is a natural candidate for a solution of our problem. It turns out that this strategy is successful.

Theorem 1.1. Suppose $\Omega \subset \mathbb{R}^{n}$ is an open set with smooth boundary, such that $\Omega^{c}$ is bounded. For $p>1$, let $v^{(p)} \in \dot{W}^{1, p}\left(\mathbb{R}^{n}\right)$ solve

$$
\operatorname{div}\left(\left|\nabla v^{(p)}\right|^{p-2} \nabla v^{(p)}\right)=0 \quad \text { in } \Omega,
$$

and $v^{(p)}=1$ on $\Omega^{c}$. Then

$$
(1-p) \log v^{(p)} \rightarrow u
$$

locally uniformly in $\bar{\Omega}$, where $u \in C_{\mathrm{loc}}^{0,1}(\bar{\Omega})$ is a proper weak solution of $(1)$ and $(2)$.

This theorem can be interpreted as a result on the behaviour of special $p$-harmonic functions (namely the ones giving the $p$-capacity of $\Omega^{c}$ ) as $p$ tends to 1 . But of course it also implies in particular that a weak solution of the inverse mean curvature flow exists. The proof turns out to be quite simple and direct. In addition to the stated facts, it also gives a gradient bound and an estimate for the growth of $u$ at infinity. A maximum principle and a comparison principle (for solutions constructed with this method) follow directly from the corresponding facts about $p$-harmonic functions. All of this, however, has already been 
proved for proper weak solutions of the inverse mean curvature flow by Huisken-Ilmanen [4], in the case of the gradient estimate even a slightly better result.

The method we use gives a link between two problems of different types: the inverse mean curvature flow on the one hand, which is parabolic and not a variational problem, and $p$-harmonic functions on the other hand, which are solutions of an archetypal elliptic variational problem. Moreover, we obtain a construction of solutions of (1) and (2) with elliptic rather than parabolic methods, which may be helpful when equation (1) is studied independently of the inverse mean curvature flow, as a problem in its own right.

\section{Construction of the solutions}

In this section we give the proof of Theorem 1.1. Let thus $\Omega \subset \mathbb{R}^{n}$ be open with smooth boundary, such that $\Omega^{c}$ is bounded. We denote the open ball in $\mathbb{R}^{n}$ with centre $x_{0}$ and radius $r$ by $B_{r}\left(x_{0}\right)$. Let $R>0$ be the supremum of all numbers $r>0$ such that each $x \in \partial \Omega$ is on a sphere $\partial B_{r}\left(x_{0}\right)$ with $B_{r}\left(x_{0}\right) \subset \Omega^{c}$.

Fix $p>1$, and suppose that $v \in \dot{W}^{1, p}\left(\mathbb{R}^{n}\right)$ is a minimizer of the functional

$$
E_{p}(w)=\frac{1}{p} \int_{\mathbb{R}^{n}}|\nabla w|^{p} d x
$$

among all $w \in \dot{W}^{1, p}\left(\mathbb{R}^{n}\right)$ with $w \geq 1$ in $\Omega^{c}$. Then $v$ solves equation 5 with boundary data $v=1$ on $\partial \Omega$. If $B_{r}\left(x_{0}\right) \subset \Omega^{c}$, the function

$$
w(x)=\left(\frac{\left|x-x_{0}\right|}{r}\right)^{(n-p) /(1-p)}
$$

is another solution of (5) with $w \leq 1$ on $\partial \Omega$. Since the equation is subject to a comparison principle (see, e.g., Tolksdorf [9]), we have

$$
v(x) \geq\left(\frac{\left|x-x_{0}\right|}{r}\right)^{(n-p) /(1-p)}, \quad x \in \Omega .
$$

Similarly, if $B_{S}\left(y_{0}\right)$ is a ball with $\Omega^{c} \subset B_{S}\left(y_{0}\right)$, we conclude

$$
v(x) \leq\left(\frac{\left|x-y_{0}\right|}{s}\right)^{(n-p) /(1-p)}, \quad x \in \Omega \backslash\left\{y_{0}\right\} .
$$

According to the results of Lewis [6], we have $v \in C_{\text {loc }}^{1, \alpha}(\Omega)$ for some $\alpha>0$ (depending on $n$ and $p$ ). Since $\partial \Omega$ is smooth, we can even show that $v \in C_{\mathrm{loc}}^{1, \alpha}(\bar{\Omega})$ by the application of a reflection principle and arguments as in [6].

Now let $B_{r}\left(x_{0}\right) \subset \Omega$ be a fixed ball. With arguments from J. Moser [7] (which are easily adapted to our situation) or with other standard arguments, we prove the Harnack inequality

$$
\sup _{B_{r / 2}\left(x_{0}\right)} v \leq C_{1} \inf _{B_{r / 2}\left(x_{0}\right)} v
$$


for a certain constant $C_{1}$ that depends only on $n$ and $p$. If $\eta \in C_{0}^{\infty}(\Omega)$ is a cut-off function, we compute

$$
\begin{aligned}
\int_{\Omega} \eta^{p}|\nabla v|^{p} d x & =-p \int_{\Omega} \eta^{p-1} v|\nabla v|^{p-2} \nabla v \cdot \nabla \eta d x \\
& \leq p\left(\int_{\Omega} \eta^{p}|\nabla v|^{p} d x\right)^{(p-1) / p}\left(\int_{\Omega} v^{p}|\nabla \eta|^{p} d x\right)^{1 / p} .
\end{aligned}
$$

Thus

$$
\int_{\Omega} \eta^{p}|\nabla v|^{p} d x \leq p^{p} \int_{\Omega} v^{p}|\nabla \eta|^{p} d x .
$$

Together with the Harnack inequality this gives

$$
r^{p-n} \int_{B_{r / 4}\left(x_{0}\right)}|\nabla v|^{p} d x \leq C_{2} \inf _{B_{r / 2}\left(x_{0}\right)} v^{p}
$$

for a constant $C_{2}$ that depends only on $n$ and $p$. Now we apply the results of Lewis [6] again. They imply the existence of a constant $C_{3}$, depending on $n$ and $p$, such that

$$
\sup _{B_{r / 8}\left(x_{0}\right)}|\nabla v| \leq \frac{C_{3}}{r} \inf _{B_{r / 2}\left(x_{0}\right)} v .
$$

In particular we have

$$
\lim _{|x| \rightarrow \infty} \frac{|\nabla v|}{v}=0
$$

Next we define

$$
u=(1-p) \log v
$$

If $B_{r}\left(x_{0}\right) \subset \Omega^{c}$, we have

$$
u(x) \leq(n-p) \log \left(\frac{\left|x-x_{0}\right|}{r}\right), \quad x \in \Omega,
$$

and if $\Omega^{c} \subset B_{s}\left(y_{0}\right)$,

$$
u(x) \geq(n-p) \log \left(\frac{\left|x-y_{0}\right|}{s}\right), \quad x \in \Omega \backslash\left\{y_{0}\right\} .
$$

We know that $u \in C_{\mathrm{loc}}^{1, \alpha}(\bar{\Omega})$ and

$$
\lim _{|x| \rightarrow \infty}|\nabla u|=0 .
$$

Most importantly, $u$ satisfies equation (4) and $u=0$ on $\partial \Omega$.

Inequality (6) together with the definition of $R$ implies

$$
|\nabla u| \leq \frac{n-p}{R} \quad \text { on } \partial \Omega .
$$


Thus for every $\beta>(n-p) / R$, the set

$$
\Omega_{\beta}=\{x \in \Omega:|\nabla u(x)|>\beta\}
$$

is a bounded, open set with $\bar{\Omega}_{\beta} \cap \partial \Omega=\emptyset$. On $\partial \Omega_{\beta}$, we have $|\nabla u|=\beta$.

Differentiating equation (4), we obtain

$$
\operatorname{div}\left[|\nabla u|^{p-2} \nabla u_{x_{i}}+(p-2)|\nabla u|^{p-4}\left(\nabla u \cdot \nabla u_{x_{i}}\right) \nabla u\right]=p|\nabla u|^{p-2} \nabla u \cdot \nabla u_{x_{i}}
$$

for $i=1, \ldots, n$, at least in $\Omega_{\beta}$. Thus

$$
\begin{aligned}
& \operatorname{div}\left[|\nabla u|^{p-2} u_{x_{i}} \nabla u_{x_{i}}+(p-2)|\nabla u|^{p-4} u_{x_{i}}\left(\nabla u \cdot \nabla u_{x_{i}}\right) \nabla u\right] \\
& \quad=p|\nabla u|^{p-2} u_{x_{i}} \nabla u \cdot \nabla u_{x_{i}}+|\nabla u|^{p-2}\left|\nabla u_{x_{i}}\right|^{2}+(p-2)|\nabla u|^{p-4}\left(\nabla u \cdot \nabla u_{x_{i}}\right)^{2} .
\end{aligned}
$$

For the function $f=|\nabla u|^{2}$, this means

$$
\operatorname{div}\left[\frac{1}{p} \nabla f^{p / 2}+\left(\nabla u \cdot \nabla f^{p / 2-1}\right) \nabla u\right]-\nabla u \cdot \nabla f^{p / 2} \geq \frac{p-1}{4} f^{p / 2-2}|\nabla f|^{2} .
$$

If we write

$$
A=\mathrm{id}+(p-2) \frac{\nabla u \otimes \nabla u}{f}
$$

the last inequality is equivalent to

$$
\operatorname{div}\left(f^{p / 2-1} A \nabla f\right)-p f^{p / 2-1} \nabla u \cdot \nabla f \geq \frac{p-1}{4} f^{p / 2-2}|\nabla f|^{2} .
$$

Since $p>1$, the matrix $A$ is uniformly positive definite. Regarding $f^{p / 2-1} A$ and $-p f^{p / 2-1} \nabla u$ as the coefficients of a linear partial differential operator, we find that this operator is elliptic and subject to a maximum principle. Hence

$$
\sup _{\Omega_{\beta}} f \leq \sup _{\partial \Omega_{\beta}} f \leq \beta^{2} .
$$

We conclude that

$$
\sup _{\bar{\Omega}}|\nabla u| \leq \frac{n-p}{R} .
$$

We also note that $u$ is a minimizer of the functional

$$
J_{u}^{p}(w ; K)=\int_{K}\left(\frac{1}{p}|\nabla w|^{p}+w|\nabla u|^{p}\right) d x
$$

for every precompact set $K \subset \Omega$ in the sense that

$$
J_{u}^{p}(u ; K) \leq J_{u}^{p}(w ; K)
$$


whenever $w \in W_{\text {loc }}^{1, p}(\Omega)$ satisfies $w=u$ in $\Omega \backslash K$. Indeed, for every such $w$, we have, by (4),

$$
\begin{aligned}
\int_{K}(u-w)|\nabla u|^{p} d x & =\int_{\Omega}|\nabla u|^{p-2} \nabla u \cdot(\nabla w-\nabla u) d x \\
& \leq \frac{1}{p} \int_{K}\left(|\nabla w|^{p}-|\nabla u|^{p}\right) d x
\end{aligned}
$$

It is now easy to complete the proof of Theorem 1.1 If $v^{(p)} \in \dot{W}^{1, p}\left(\mathbb{R}^{n}\right)$ are solutions of 5. with $v^{(p)}=1$ in $\Omega^{c}$, they coincide in $\Omega$ with the functions considered above, for the solutions are unique. Thus we have uniform gradient bounds for the functions $u^{(p)}=(1-p) \log v^{(p)}$, namely

$$
\left|\nabla u^{(p)}\right| \leq \frac{n-p}{R} \quad \text { in } \bar{\Omega}
$$

There exists a sequence $p_{k} \rightarrow 1$ such that $u^{\left(p_{k}\right)} \rightarrow u$ locally uniformly in $\bar{\Omega}$ for a function $u \in C_{\mathrm{loc}}^{0,1}(\bar{\Omega})$. With practically the same arguments that were used in [4] to prove a compactness result for weak solutions of (1), we can show that $u$ is a weak solution of (1). We include a version of these arguments below for completeness. First, however, we note the following: Once it is proved that $u$ is a weak solution of (1), we see that it is proper because of (7). Another result of [4] states that proper weak solutions of the problem are unique. Thus $u$ does not depend on the choice of $p_{k}$, and we have in fact $u^{(p)} \rightarrow u$ locally uniformly in $\bar{\Omega}$ as $p \rightarrow 1$.

It remains to show that $(3)$ holds for every precompact set $K \subset \Omega$ and every $w \in$ $C_{\text {loc }}^{0,1}(\Omega)$ with $w=u$ in $\Omega \backslash K$. Suppose such a set $K$ and such a function $w$ are given. Choose $\eta \in C_{0}^{\infty}(\Omega)$ with $0 \leq \eta \leq 1$ and $\eta \equiv 1$ in $K$, and insert $\eta w+(1-\eta) u^{(p)}$ as a test function into $J_{u^{(p)}}^{p}(\cdot ; \operatorname{supp} \eta)$ in inequality $[8$. . It follows that

$$
\begin{aligned}
\int_{\operatorname{supp} \eta} & \left(\frac{1}{p}\left|\nabla u^{(p)}\right|^{p}+\eta\left(u^{(p)}-w\right)\left|\nabla u^{(p)}\right|^{p}\right) d x \\
\leq & \frac{1}{p} \int_{\operatorname{supp} \eta}\left|\eta \nabla w+(1-\eta) \nabla u^{(p)}+\left(w-u^{(p)}\right) \nabla \eta\right|^{p} d x \\
\leq & \frac{3^{p-1}}{p} \int_{\operatorname{supp} \eta}\left(\eta^{p}|\nabla w|^{p}+(1-\eta)^{p}\left|\nabla u^{(p)}\right|^{p}+\left|w-u^{(p)}\right|^{p}|\nabla \eta|^{p}\right) d x .
\end{aligned}
$$

Choosing first $w=u$ and letting $p \rightarrow 1$, we obtain

$$
\int_{\Omega} \eta|\nabla u| d x \geq \limsup _{k \rightarrow \infty} \int_{\Omega} \eta\left|\nabla u^{\left(p_{k}\right)}\right|^{p_{k}} d x,
$$

and we infer $\left|\nabla u^{\left(p_{k}\right)}\right|^{p_{k}} \rightarrow|\nabla u|$ in $L_{\text {loc }}^{1}(\Omega)$. Considering $(9)$ again, we conclude that 3 , holds. This completes the proof of Theorem 1.1 . 


\section{References}

[1] Gerhardt, C.: Flow of nonconvex hypersurfaces into spheres. J. Differential Geom. 32, 299_ 314 (1990) Zbl 0708.53045 MR 1064876

[2] Huisken, G., Ilmanen, T.: A note on the inverse mean curvature flow. In: Proc. Workshop on Nonlinear Part. Diff. Equ. (Saitama Univ.) (1997)

[3] Huisken, G., Ilmanen, T.: The Riemannian Penrose inequality. Int. Math. Res. Not. 1997, no. 20, 1045-1058 Zbl 0905.53043 MR 1486695

[4] Huisken, G., Ilmanen, T.: The inverse mean curvature flow and the Riemannian Penrose inequality. J. Differential Geom. 59, 353-437 (2001) Zbl 1055.53052 MR 1916951

[5] Huisken, G., Ilmanen, T.: Higher regularity of the inverse mean curvature flow. Preprint (2002)

[6] Lewis, J. L.: Regularity of the derivatives of solutions to certain degenerate elliptic equations. Indiana Univ. Math. J. 32, 849-858 (1983) Zbl 0554.35048 MR 0721568

[7] Moser, J.: On Harnack's theorem for elliptic differential equations. Comm. Pure Appl. Math. 14, 577-591 (1961) Zbl 0111.09302 MR 0159138

[8] Smoczyk, K.: Remarks on the inverse mean curvature flow. Asian J. Math. 4, 331-335 (2000) Zbl 0989.53040 MR 1797584

[9] Tolksdorf, P.: On the Dirichlet problem for quasilinear equations in domains with conical boundary points. Comm. Partial Differential Equations 8, 773-817 (1983) Zbl 0515.35024 MR 0700735

[10] Urbas, J. I. E.: On the expansion of starshaped hypersurfaces by symmetric functions of their principal curvatures. Math. Z. 205, 355-372 (1990) Zbl 0691.35048 MR 1082861 